\title{
19. Soggettività e oggettività negli articoli a carattere informativo di tre giornali italiani
}

Maria Tell

Stockholms universitet

\section{Introduzione}

Questo studio mira ad esaminare come soggettività e oggettività vengono espresse in alcuni articoli di giornale a carattere informativo, quindi non in articoli editoriali. Partendo dal presupposto che il linguaggio giornalistico non è mai del tutto neutro, mi interessa osservare con quali modalità gli autori degli articoli, con l'aiuto della lingua, intendono esprimere soggettività, oggettività ed opinioni ideologiche. Roger Fowler ha individuato e descritto la presenza di un fattore ideologico in ogni tipo di linguaggio giornalistico:

News is a representation of the world in language; because language is a semiotic code, it imposes a structure of values, social and economic in origin, on whatever is represented; and so inevitably news, like every discourse, constructively patterns that of which it speaks. News is a representation in this sense of construction; it is not a value-free reflection of 'facts'. [...] There are always different ways of saying the same thing, and they are not random, accidental alternatives. Differences in expression carry ideological distinctions (and thus differences in representation) (Fowler I99I: 4).

Il corpus preso in considerazione in questo studio consiste di articoli che trattano delle manifestazioni svoltesi a Roma contro Silvio Berlusconi in seguito alle sue dimissioni nel novembre 20II. Si è scelto di prendere in considerazione tre dei principali quotidiani italiani, ognuno con un'impronta ideologica diversa: l'Unità, di sinistra, il Corriere della Sera, liberale, ed il Giornale, di destra e oltretutto di proprietà della famiglia Berlusconi. L'analisi mira a mettere in evidenza le modalità con cui soggettività e

Come citare questo capitolo:

Tell, Maria, Soggettività e oggettività negli articoli a carattere informativo di tre giornali italiani. In: Engwall, Gunnel \& Fant, Lars (eds.) Festival Romanistica. Contribuciones lingüísticas - Contributions linguistiques - Contributi linguistici - Contribuições linguísticas. Stockholm Studies in Romance Languages. Stockholm: Stockholm University Press. 20I 5, pp. 360-376. DOI: http://dx.doi.org/Io.I6993/bac.s. License: CC-BY 
oggettività si manifestano nei rispettivi articoli che troviamo pubblicati sui siti internet dei giornali. Come punto di partenza dello studio verranno applicate soprattutto le teorie dell'analisi del discorso. L'analisi linguistica si ispira all'analisi critica del discorso così com'è stata elaborata in principio da Norman Fairclough, secondo il quale i discorsi sono

ways of representing the world. [...] Different discourses are different perspectives of the world, and they are associated with the different relations people have to the world, which in turn depends on their positions in the world, their social and personal identities [...]. Discourses not only represent the world as it is (or rather is seen to be), they are also projective, imaginaries, representing possible worlds which are different from the actual world, and tied into projects to change the world in particular directions (Fairclough 2003: I 24).

Un vantaggio delle teorie di Fairclough è che queste si focalizzano sia sull'aspetto ideologico di diversi discorsi, sia sul modo in cui questo aspetto viene espresso nel linguaggio attraverso l'uso di vari procedimenti linguistici. Inoltre in queste si distingue tra un ordine del discorso più generale, che tratta argomenti pertinenti allo stesso campo (ad esempio i media, le università o i servizi sanitari), e diversi discorsi contrastanti all'interno dell'ordine del discorso generale in questione (I995: 55-56). In questo caso si ha un ordine del discorso giornalistico all'interno del quale diversi discorsi ideologici, uno di sinistra, uno liberale e uno di destra, presentano la loro immagine della realtà. L'analisi linguistica di Fairclough prende spunto innanzitutto dalla grammatica sistemico-funzionale di M.A.K. Halliday, che si focalizza sulle funzioni linguistiche coinvolte nel processo di creazione del senso nel linguaggio. Secondo Halliday lo scopo dell'analisi sistemica di un testo è quello di spiegare gli effetti da questo ottenuti: come e perché un testo suscita una certa impressione e perviene al suo significato $(2004: 658)^{\mathrm{I}}$. Ciò che sarà analizzato più a fondo nello studio presente sono i diversi mezzi linguistici utilizzati dai giornalisti nelle frasi informative al fine di esprimere soggettività, oggettività ed opinioni ideologiche. Questi fenomeni si possono osservare, per esempio, nell'uso di parole con connotazioni positive o negative, nell'impiego della transitività, della nominalizzazione e della modalità, e nell'uso di varie figure retoriche (Fowler I991: 64-79; Fairclough I995: 26-27).

\section{Soggettività e oggettività negli articoli scelti}

Tutti e tre gli articoli di giornale selezionati per questo studio trattano in gran parte degli stessi eventi: i manifestanti che festeggiano le 
dimissioni di Silvio Berlusconi con lo spumante, il lancio di monetine contro Berlusconi da parte dei manifestanti, l'arrivo e/o la partenza di Berlusconi a/da Palazzo Chigi e la presenza dell'orchestra, la Resistenza Musicale Permanente, che suona per celebrare le dimissioni del premier. L'obiettivo di questa analisi è osservare se vi siano delle differenze o delle similitudini linguistiche nella descrizione di questi eventi sui giornali, e se vi siano inoltre delle differenze nella scelta dell'informazione presentata al lettore. Nel caso di interviste e di citazioni ci si aspetterebbe infatti che il giornalista facesse emergere diversi punti di vista ed opinioni contrastanti per mantenere l'oggettività e l'equilibrio dell'informazione. Come affermato da Fairclough, rappresentazioni divergenti dello stesso evento possono dipendere dallo scopo, dai valori e dalle priorità della comunicazione e, nell'analisi testuale, queste differenze sono spesso osservabili attraverso l'uso di discorsi diversi (I995: 4I). Si è partiti con un'analisi dei titoli dei giornali, i quali trasmettono l'informazione ritenuta la più importante e che è posta dunque in primo piano (si veda tra gli altri Fairclough 1995: I I9). I titoli saranno di seguito citati per intero mentre per quanto riguarda l'analisi degli articoli, per motivi di spazio, ho scelto dei brani rappresentativi (correndo così il rischio di aver fatto delle scelte soggettive inconsce, come succede ad ogni autore).

\section{il Giornale:}

Berlusconi consegna le dimissioni al Colle. E dall'Italia dell'odio sputi, monete e grida.

Una folla inferocita ha seguito tutti gli spostamenti di Silvio Berlusconi da Palazzo Chigi al Quirinale fino a Palazzo Grazioli. Caroselli, orchestre, Bella Ciao e cartelli che inneggiano alla Liberazione. FOTO-VIDEO. La folla inferocita assedia i palazzi del potere: prima è festa e poi la vendetta. Urla, sputi e insulti. E Di Pietro fa il gesto dell'ombrello.

di Francesco Maria Del Vigo

Il Cavaliere è salito al Colle e ha fatto un passo indietro FOTO VIDEO ${ }^{2}$

E Di Pietro fa il gesto dell'ombrello

\section{l'Unità:}

Bandiere, musica, cori ma anche monetine per l'addio del premier di Natalia Lombardo

Corriere della Sera:

FESTEGGIAMENTI FINO A TARDA NOTTE

“Bye Bye Berlusconi”, festa di piazza 
Traffico in tilt: migliaia di persone davanti al Quirinale e Palazzo Grazioli prima e dopo dimissioni [sic!] del premier. Il Popolo Viola: "Gli abbiamo tirato le monetine".

[Redazione online]

Si nota immediatamente come il titolo de il Giornale sia molto più lungo degli altri due e includa anche un piccolo sommario relativo al contenuto dell'articolo. Sono presenti delle ripetizioni di frasi e di parole che sottolineano il messaggio e cercano di accreditare la pretesa di veridicità di ciò che è detto (come è noto, più una cosa viene ripetuta e più facilmente viene accettata come vera). Nel titolo de il Giornale il reporter riesce ad esprimere alcuni pareri soggettivi, soprattutto attraverso la scelta di nomi, verbi ed aggettivi che trasmettono connotazioni positive quando si parla di Berlusconi e connotazioni negative invece nei confronti dei manifestanti. Una divisione delle funzioni degli elementi della frase, basata sulla teoria di Halliday (2004: I77-I82), ci permette di distinguere meglio la costruzione delle frasi e gli elementi soggettivi inseriti dal reporter, quali la connotazione e la combinazione di attributi e verbi:

\section{Tabella 1.}

\begin{tabular}{lllll}
\hline Agente & Processo & Scopo & Paziente & Circostanze \\
\hline Berlusconi & consegna & le dimissioni & & al Colle \\
$\begin{array}{l}\text { Una folla } \\
\text { inferocita }\end{array}$ & ha seguito & $\begin{array}{l}\text { tutti gli } \\
\text { spostamenti di }\end{array}$ & $\begin{array}{l}\text { Silvio } \\
\text { Berlusconi }\end{array}$ & $\begin{array}{l}\text { da Palazzo } \\
\text { Chigi [...] fino a } \\
\text { Palazzo Grazioli }\end{array}$ \\
$\begin{array}{l}\text { La folla } \\
\text { inferocita }\end{array}$ & \multirow{2}{*}{ assedia } & $\begin{array}{l}\text { il palazzo del } \\
\text { potere }\end{array}$ & & \\
\hline
\end{tabular}

Il reporter scrive che Berlusconi "consegna" le dimissioni invece di usare l'espressione più comune "dare le dimissioni”" (risultato di una ricerca su Google: I I 70 ooo risultati per la prima versione e 2990 ○o per la seconda; nello Zingarelli si trova solo l'espressione con il verbo dare). Le connotazioni del verbo consegnare suggeriscono l'immagine che Berlusconi stia regalando o sacrificando qualcosa, elemento a sua volta rafforzato dalla frase "ha fatto un passo indietro". Nelle descrizioni dei manifestanti prevalgono invece nomi ed aggettivi con connotazioni negative. Si osservi la frase in cui è riportata la risposta dei manifestanti alle dimissioni del premier, “e dall'Italia dell'odio 
sputi, monete e grida", ripresa poi in seguito da un'altra frase, "Urla', sputi e 'insulti", e inoltre anche dall'uso della parola "vendetta”. Tutte queste parole presenti nelle frasi citate rappresentano attività aggressive e negative. È da notare anche l'espressione "folla inferocita", che appare due volte, dove l'attributo "inferocita", insieme ad un'anonima "folla" quale agente, dà l'impressione di una forza feroce, incontrollabile e intimidante. Proseguiamo con la costruzione delle frasi "Una folla inferocita ha seguito tutti gli spostamenti di Berlusconi..." e "la folla inferocita assedia il palazzo di potere"; qui la folla è in posizione di agente, è responsabile dell'azione ed è accompagnata da un verbo come assediare, che riporta ad un contesto di aggressività e guerra e che, in associazione con la "folla", rinforza il parere negativo del giornalista nei confronti dei manifestanti. Berlusconi, a sua volta, è posto in posizione di paziente, è il partecipante agito e viene rappresentato come una vittima perseguitata dai manifestanti, che ricoprono la parte dei persecutori. Il giornalista inoltre mette in risalto un gesto osceno di Antonio Di Pietro, che appartiene alla fazione politica opposta a Berlusconi.

Dunque, già nel titolo traspare l'atteggiamento soggettivo e negativo del reporter nei confronti dei manifestanti antiberlusconiani, da una parte attraverso l'uso di diversi procedimenti linguistici quali la prevalenza e la ripetizione di parole e di frasi con connotazioni negative nella descrizione dei manifestanti, e dall'altra per la mancanza di una loro individualizzazione. Su nove frasi ne sono state individuate otto soggettive ed una sola oggettiva: "caroselli, orchestre, Bella ciao e cartelli che inneggiano alla Liberazione”.

Rispetto al titolo de il Giornale ed anche a quello del Corriere della Sera, il titolo de l'Unità è molto più breve e conciso. È anche piuttosto oggettivo: le parole usate sono neutre e non sono riportati pareri sul contenuto da parte della giornalista. Inizia focalizzandosi sulla gioia dei manifestanti che festeggiano. C'è un certo distacco dalla figura di Berlusconi da parte dell'autore, che si concentra piuttosto sul fatto che si stia allontanando dal potere, vale a dire sull'effetto delle dimissioni e non sulle stesse che, al contrario, non vengono menzionate. Inoltre la giornalista per designare Berlusconi usa solo il titolo formale, “premier", e non il nome proprio.

L'articolo del Corriere della Sera è l'unico a non essere firmato da un giornalista in particolare ma da un'anonima redazione online. Perciò, non potendo attribuire l'articolo ad una persona specifica, designerò la persona o le persone responsabili con il termine più generico di emittente del testo. 
Nel Corriere della Sera il titolo si focalizza sull'atmosfera di festa e sul punto di vista dei manifestanti. Come è stato osservato, le scelte compiute circa gli elementi inseriti volutamente nel testo e quelli invece esclusi possono essere rilevanti per quanto riguarda l'analisi degli aspetti ideologici (Fairclough I995: I05-07). Né qui né nell'articolo de l'Unità vengono menzionati gli sputi, gli insulti e le urla della folla inferocita che l'emittente de il Giornale ha invece scelto di mettere in risalto. In quest'ultimo, all'interno del catenaccio, l'emittente trasmette informazioni oggettive sulla situazione del traffico nel centro di Roma, notizia che gli altri due giornali non menzionano affatto. Supponendo che un articolo giornalistico inizi con il trasmettere l'informazione ritenuta la più importante, si può dedurre che uno degli scopi primari dell'emittente fosse quello di mettere al corrente il cittadino medio sulle conseguenze delle manifestazioni. Non a caso infatti l'ultimo paragrafo dell'articolo tratta solamente dei problemi del traffico nella capitale.

Anche il resto del titolo è di carattere oggettivo: vengono menzionate le dimissioni del premier e la presenza di manifestanti senza però esprimere giudizi od opinioni personali, attraverso un linguaggio privo di connotazioni particolari. Il lancio delle monetine viene presentato tramite il discorso diretto, riportato attraverso la citazione delle parole di uno dei membri del Popolo Viola, senza alcun commento da parte dall'emittente, che mantiene dunque un approccio oggettivo preferendo lasciar parlare i fatti e le persone. Un'altra differenza tra il Giornale da una parte e l'Unità e il Corriere della Sera dall'altra è che nei titoli di questi ultimi vi sono solamente frasi nominali, strutture usate assai frequentemente nel linguaggio giornalistico italiano; in quest'ottica il linguaggio de il Giornale costituisce dunque un'eccezione.

\section{L'articolo de il Giornale}

L'articolo si caratterizza fin dall'inizio per l'assai frequente ripetizione di nomi e verbi con connotazioni negative a caratterizzare i manifestanti, come abbiamo già visto nell'analisi dei titoli, ma anche di domande e di figure retoriche, di metafore, di frasi affermative e giudizi che ribadiscono la volontà di discreditare i manifestanti. Si veda ad esempio il paragrafo seguente, che inizia e termina con una ripetizione di nomi e di verbi che sottolineano l'aggressività dei manifestanti, come sputi, sputare, insulti, insultare, assediare, urlare, lanciare monetine:

Insulti, sputi e poi la festa. Cosa c'entrano i cori da stadio con una giornata come questa? Il morso della crisi si avvicina all'osso del Paese, la 
politica annaspa ma c'è qualcuno che ci vede qualcosa di buono. Non è la solita storia del bicchiere mezzo pieno e mezzo vuoto, questa volta il calice è prosciugato. Ma per gli sfascisti, quelli con la bava alla bocca che vogliono solo la testa del Cavaliere, il miraggio dell'oasi antiberlusconiana ora è realtà. E quindi saltano i tappi e si alzano i cori [...]. Alla faccia di tutto, anche del buon gusto e della razionalità. Ora tocca al revanscismo, lo aspettavano da anni: è l'Italia che si dice indignata perché è politicamente scorretto essere incazzati. Eppure sputano, urlano e lanciano monetine. Insultano qualunque membro del governo abbiano a tiro e assediano i palazzi del potere.

La ricorrenza di questi nomi e verbi ha come effetto quello di sottolineare il comportamento selvaggio e immotivato dei manifestanti, visto attraverso gli occhi del reporter. Queste ripetizioni non solo creano una coesione lessicale con connotazioni negative (per quanto riguarda la coesione lessicale si veda Halliday 2004: 57 I-575), ma costituiscono inoltre un esempio di ciò che Fairclough definisce come "discoursal overkill”, cioè una coesione che porta ad effetti esagerati (I995: IOI). Per quanto riguarda le domande e le figure retoriche, si può notare che il reporter fa uso di tali procedimenti al fine di mettere ulteriormente in risalto l'irresponsabilità dei manifestanti e la gravità della crisi politica attuale, accompagnati da un tono di forte indignazione. Si veda infatti la domanda retorica "Cosa c'entrano i cori da stadio con una giornata come questa?", la cui risposta è sottintesa: non c'entrano affatto. Il brano prosegue con metafore drammatiche tese anch'esse a sottolineare la gravità della situazione. L'uso di verbi all'indicativo in queste espressioni metaforiche ha la funzione di confermare la veridicità dell'enunciato del reporter, essendo il modo verbale che generalmente si usa per indicare un fatto come reale, vero: "il morso della crisi si avvicina", "la politica annaspa", "qualcuno ci vede qualcosa di buono", "il calice è prosciugato", "vogliono solo la testa del cavaliere", "tocca al revanscismo”, ecc. Il reporter ricorre più volte all'uso della congiunzione avversativa ma per contrappore la crisi politica attuale all'irresponsabilità dei manifestanti: "Il morso della crisi si avvicina, la politica annaspa ma c'è qualcuno che ci vede qualcosa di buono", "questa volta il calice è prosciugato. Ma per gli sfascisti [...] il miraggio dell'oasi antiberlusconiana ora è realtà" (s.n.). Inoltre, per descrivere i manifestanti vengono utilizzati l'attributo "sfascisti" e l'espressione figurata "la bava alla bocca", elementi entrambi molto negativi e dispregiativi. Ancora una volta, tramite procedimenti linguistici quali la scelta dei nomi, degli attributi, delle apposizioni e dei verbi, i manifestanti sono presentati 
come degli aggressori e Berlusconi come una vittima. Questi procedimenti hanno qui una funzione di orientazione, servono infatti a guidare le simpatie del lettore nei confronti dei protagonisti (per la funzione di orientazione si veda Fairclough I995: 92). In questo caso le simpatie del reporter sono indubbiamente dirette verso Silvio Berlusconi e il suo governo.

L'articolo prosegue dunque secondo queste modalità, per mezzo di procedimenti linguistici che esprimono la soggettività del reporter che rimprovera i manifestanti e allo stesso tempo difende Berlusconi. Solamente uno dei paragrafi dell'articolo è piuttosto oggettivo e descrive le manifestazioni:

Fuori dalla Camera, la festa è iniziata con il "Bye, Bye Silvio, Party...?" del Popolo Viola un circo ambulante che segue il Cavaliere da Palazzo Chigi a Montecitorio fino al Quirinale. "Oggi - ha scritto il blogger Viola Gianfranco Mascia - è il grande giorno. Questo I2 novembre ce lo segneremo nel calendario come il giorno della Liberazione”. In piazza Colonna suona un'orchestrina, per strada intonano Bella Ciao e sotto al Quirinale la "Resistenza musicale permanente" si è data appuntamento per eseguire l'“Hallelujah dal Messiah” [sic!] di Handel.

Qui il giornalista non commenta la citazione del rappresentante del Popolo Viola e mancano avverbi, attributi, apposizioni e complementi a determinare verbi e nomi. Il linguaggio è neutro, senza connotazioni particolari, con l'eccezione dell'apposizione "circo ambulante" per descrivere i Viola. L'ultimo paragrafo, invece, riprende il discorso dei paragrafi precedenti sull'irresponsabilità dei manifestanti, sulla gravità della crisi e sulla vittimizzazione di Berlusconi, usando gli stessi procedimenti linguistici visti in precedenza. Si può notare inoltre come la maggior parte delle frasi siano espressive e non assertive, cosa che ci si aspetterebbe invece in un articolo a carattere informativo. Ricordiamo qui la distinzione di John Searle tra frasi espressive, che esprimono sentimenti e atteggiamenti, e frasi assertive, che rappresentano le cose così come stanno (I979: viii).

\section{L'articolo de l'Unità}

Qui la giornalista passa direttamente dal titolo all'atmosfera di festa, trasmettendo l'evento dal punto di vista dei manifestanti; ciò è osservabile nella tematizzazione e nelle connotazioni dei nomi, dei verbi e degli attributi scelti nelle funzioni di agente e processo: 
"Alleluja! Alleluja! Allelujaaa”. È con il coro degli orchestrali di Santa Cecilia, il gruppo di "Resistenza Musicale Permanente", seduto con violini, archi e fiati nella piazza davanti al Quirinale, che si celebra la "festa della liberazione". I2 novembre, una data "da non dimenticare", neppure per i bambini assiepati davanti alle transenne che chiudono Palazzo Chigi o per la massa accorsa sul Colle pronta a stappare le bottiglie di spumante al passaggio di Silvio Berlusconi. Il quale ora evita gli amati bagni di folla narcisistici.

Rispetto a il Giornale, la descrizione dell'orchestra è qui molto più dettagliata e se ne sottolinea l'alto livello culturale. Le espressioni "festa della liberazione" e "da non dimenticare" sono incastrate nel discorso della reporter e non vengono attribuite a nessuno dei protagonisti, ma al contrario rimangono anonime. Qui manca ciò che Fairclough chiama framing, ovvero quegli elementi del discorso dell'emittente che circondano il discorso riportato, quali i verbi, come dice o afferma (I995: 83). Quando le citazioni sono incastrate e anonime, come in questo caso, diventa più difficile distinguere tra il discorso della giornalista e quello di altre voci. In questo modo le citazioni entrano piuttosto a far parte delle frasi affermative della giornalista che, con questo procedimento, tende ad esprimere soggettività e a riprendere il punto di vista dei manifestanti. Anche l'uso della terza persona singolare "si celebra" invece di "loro celebrano" tende al soggettivo, dal momento che può includere tutti e dunque alludere anche al "noi". Nei confronti di Berlusconi la giornalista esprime un parere negativo tramite l'uso del sarcasmo, osservabile negli aggettivi con funzione attributiva riportati nella frase "ora evita gli amati bagni di folla narcisistici”, in cui ridicolizza il presunto egocentrismo di Berlusconi. Il narcisismo non è generalmente considerato una qualità positiva e l'uso dell'aggettivo "amati", in contrapposizione al contingente abbandono di Berlusconi di questa attività a seguito delle dimissioni, contribuisce a creare un effetto ironico. Vi sono anche altre modalità grazie alle quali la giornalista esprime un personale parere negativo nei confronti di Berlusconi, come ad esempio alcune espressioni figurate:

Dalle prime ore del pomeriggio una folla spontanea si è radunata davanti ai Palazzi: Chigi, Grazioli, il Quirinale, il triangolo di potere nel cuore di Roma. Stretti stretti su via del Corso davanti alla sede del governo in un'aria di festa e di rabbia come se scoppiasse un bubbone represso.

Qui la giornalista paragona Berlusconi e il suo governo ad un fenomeno chiaramente sgradevole, "un bubbone represso", il cui scoppio, cioè le dimissioni, genera un senso di sollievo. Si può anche notare la differenza di senso tra questo articolo e quello de il Giornale creata 
dalle diverse connotazioni degli elementi linguistici scelti nelle funzioni di agente e processo.

\section{Tabella 2.}

\begin{tabular}{lll}
\hline Agente & Processo & Circostanze \\
\hline una folla spontanea & si è radunata & davanti ai palazzi \\
\hline
\end{tabular}

Si noti la differenza di connotazione tra la scelta dell'attributo "spontanea" rispetto a quella di "inferocita" de il Giornale, e quella tra l'espressione "si è radunata" e invece "assedia" apparsa sempre ne il Giornale. Ne risulta che le scelte de l'Unità sono molto più neutre rispetto a quelle de il Giornale.

Un altro esempio di soggettività è il discorso della reporter a proposito di una fermata d'autobus: "Il segretario Neccoli annuncia che al Comune di Roma chiederà il ripristino della fermata dei bus in via del Plebiscito. Era stata tolta per non disturbare i balletti di Silvio".

La prima frase è un discorso indiretto che riporta in modo oggettivo l'annuncio di Neccoli, mentre la seconda esprime un parere un po' sprezzante nei confronti di Berlusconi da parte della giornalista che, non a caso, lo chiama solo con il nome proprio, Silvio. Si tratta di una familiarità fuori luogo in questo tipo di articoli e che risulta quindi insultante. Anche questo enunciato critica l'egocentrismo di Berlusconi, sottolineando il fatto che la fermata era stata tolta per volere suo e, per di più, per non arrecare disturbo alle sue famigerate feste.

La reporter si mostra invece positiva nei confronti dei manifestanti. Non solo trasmette le manifestazioni esclusivamente dal loro punto di vista ma vi è anche una loro individualizzazione, essendo spesso descritti con parole ed espressioni con connotazioni positive:

"Siamo qui per vederlo dimettere", la signora ben vestita, funzionaria dell'Inps, è venuta "apposta da Sapri per vederlo", e "aspettiamo che se ne vada, perché chi non vede non crede, con un tipo del genere", spiega la figlia. [...] È "gente normale" con bandiere tricolori, cittadini informati arrivati lì per esserci. "Siamo romane, aspettiamo tutti che se ne vada. Vede? ho la sciarpa arancione, come ha detto quel cantante... Vecchioni”. Anziani con la copertina di Time, giovani arrabbiati gridano "Arrestatelo", o "fuori i mafiosi dallo Stato"; più ironico il "Bye bye Berlusconi. Party?". Due donne col cartello "grazie Napolitano", sorridono: "Siamo qui armate di carta e penna, da casalinghe...". Uno scanzonato ottantenne osserva. "Io devo ancora trova' uno che l'ha votato...". 
Notiamo che la signora è "ben vestita", un attributo con connotazioni positive, ed è inoltre identificata come funzionaria dell'Inps (Istituto Nazionale Previdenza Sociale); è descritta come una brava persona con un lavoro impegnativo, e quindi il lettore può confidare nel fatto che la sua critica a Berlusconi sia motivata. Allo stesso tempo la giornalista rispetta anche se solo in parte l'oggettività degli articoli informativi, rispettando il limite tra il proprio discorso e quello dei manifestanti e non commentando le citazioni, che qui sono marcate ed attribuite ad altre persone. Il riconoscimento di questo limite da parte della reporter è un fattore importante nella questione della soggettività e dell'oggettività. Mentre le citazioni non cambiano per esempio l'ordine delle parole o il contenuto della frase, il discorso indiretto può invece trasformarli, adattandoli alla voce della reporter (Fairclough I995: 8I). Allo stesso tempo mancano però opinioni che si oppongano a quelle dei manifestanti. Si vede come i manifestanti che la giornalista sceglie di presentare siano descritti come delle brave persone, innocue, come ad esempio casalinghe e pensionati. Osserviamo come una citazione incastrata nel discorso della giornalista, "gente normale", unitamente al suo parere che si tratti di "cittadini informati”, assuma il carattere di una frase affermativa che appoggia i manifestanti e li definisce 'normali' e 'informati'. Sono, cioè, persone istruite che sanno quello che fanno, a differenza della folla inferocita descritta ne $i l$ Giornale. Si noti il modo in cui la reporter riporta i gesti dei manifestanti che, sventolando la bandiera italiana, compiono un gesto patriottico. Anche qui riscontriamo una differenza rispetto a il Giornale, che invece li accusava implicitamente di non interessarsi al destino del Paese.

La reporter, tramite il linguaggio, sembra dunque voler sminuire l'aspetto violento del lancio delle monetine da parte dei manifestanti: “Tira anche un po' aria di 'monetine', da quel lontano 1993 nel quale Craxi e la classe politica fu messa alla berlina. 'Ce l'hai le monetine?', s'informa un ragazzo arrampicato sulle transenne".

L'uso dell'avverbio aggiuntivo anche indica che il lancio delle monetine non è l'unico evento che si sta verificando; l'espressione "un po", assieme alla parola monetine tra virgolette, riscuote un effetto eufemistico, diminuendo appunto l'aggressività dell'evento. Inoltre colloca il lancio di monetine entro un contesto storico-politico. La gente ha lanciato le monetine anche nel I993. A differenza de il Giornale, qui viene meno la focalizzazione sulla personalità di Berlusconi e la presunta vendetta nei suoi confronti. Il lancio delle monetine fa piuttosto parte di un rito che si ripete ogni volta in cui un governo considerato corrotto dà le dimissioni. 
Ne il Giornale abbiamo visto come Berlusconi venga rappresentato come una vittima perseguitata da una massa inferocita, tra l'altro nelle posizioni rispettivamente di paziente e di agente, attraverso le frasi e le connotazioni dei verbi e dei nomi usati. Qui vediamo, piuttosto, il contrario: Berlusconi, quando viene messo in posizione di agente, è rappresentato come qualcuno in fuga dalle proprie responsabilità nei confronti del popolo: "L'Audi blu di Berlusconi scivola dall'uscita su piazza del Parlamento, corre su per via del Tritone ma dalla folla partono fischi e urla".

Attraverso questo procedimento sintattico l'evento diventa quindi una fuga da parte di Berlusconi, che prende l'iniziativa, invece di una caccia all'uomo da parte dei manifestanti, che qui rimangono invece inerti. Si noti che anche la scelta dei verbi scivolare e correre indica una fuga precipitosa.

\section{L'articolo del Corriere della sera}

L'articolo del Corriere della Sera, a mio parere, è il più oggettivo sia nei confronti dei manifestanti, sia nei confronti di Berlusconi e del suo governo. Il linguaggio in questo articolo non è solo più neutro ma anche molto più formale. Si veda, per esempio, il primo paragrafo, in cui è rispettato l'equilibrio tra i festeggiamenti dei manifestanti e la gravità di un evento come le dimissioni del premier.

ROMA - Più di un migliaio di persone ha festeggiato le dimissioni del premier Silvio Berlusconi in piazza del Quirinale. Dalle ore s6 di sabato, un nutrito gruppo di persone si era riunito davanti al palazzo per assistere all'ultimo atto del governo Berlusconi. Il Presidente del Consiglio, Silvio Berlusconi, è arrivato alle 20.57 per rassegnare le dimissioni nelle mani del Capo dello Stato.

Nella tabella sottostante è osservabile la mancanza di connotazioni particolari degli elementi linguistici, fattore che riconduce all'oggettività.

\section{Tabella 3.}

\begin{tabular}{|c|c|c|c|}
\hline Agente & Processo & Circostanze & Scopo \\
\hline $\begin{array}{l}\text { un nutrito } \\
\text { gruppo di } \\
\text { persone }\end{array}$ & si era riunito & $\begin{array}{l}\text { davanti al } \\
\text { palazzo }\end{array}$ & $\begin{array}{l}\text { per assistere all'ul- } \\
\text { timo atto del governo } \\
\text { Berlusconi }\end{array}$ \\
\hline $\begin{array}{l}\text { Il Presidente del } \\
\text { Consiglio, Silvio } \\
\text { Berlusconi }\end{array}$ & è arrivato & alle 20.57 & $\begin{array}{l}\text { per rassegnare le } \\
\text { dimissioni nelle mani } \\
\text { del Capo dello Stato }\end{array}$ \\
\hline
\end{tabular}


L'emittente non esprime opinioni proprie, né assume il punto di vista dell'uno o dell'altro protagonista di questi eventi. La correttezza e la veridicità dell'informazione sono sottolineate dalla menzione di orari e di luoghi esatti. I manifestanti, a differenza che ne $i l$ Giornale, sono descritti in modo neutrale, come un gruppo di persone qualsiasi, e anche Berlusconi è presentato in modo neutro e corretto, attraverso l'uso del suo titolo formale e del nome per intero. Anche i verbi riunirsi e arrivare sono neutri, privi di connotazioni particolari.

Gran parte dell'articolo prosegue poi, come ne l'Unità, descrivendo le manifestazioni e i luoghi dove si svolgono. L'emittente del Corriere della Sera è, però, più cauto e mantiene una maggiore distanza dai manifestanti rispetto alla reporter de l'Unità. Inoltre rileva aspetti sia positivi sia negativi delle manifestazioni: da una parte l'atmosfera di festa e di solidarietà e dall'altra l'affollamento ed i problemi del traffico da queste causati. Compie anche una distinzione tra manifestanti più o meno pacifici. Ne vediamo un esempio di seguito, dove l'espressione non solo, ma anche rafforza la sensazione di affollamento creato dai manifestanti:

Non solo tutta la facciata della Consulta è occupata dalla folla, ma anche il lato delle scuderie del Quirinale, con la Polizia a far da cordone. "Buffone, Buffone": le urla della folla sono intervallate dalle note di un'orchestra che nell'attesa del premier ha suonato Hallelujah e l'Inno d'Italia. Ma c'è anche chi intona "Bella ciao", seguita da "Meno male che Giorgio c'è".

A seguito vi è poi un confronto, introdotto da un $m a$ avversativo, tra l'orchestra che suona brani inoffensivi e alcune persone che cantano invece brani più controversi come Bella ciao, la canzone partigiana simbolo della seconda guerra mondiale, e "Meno male che Giorgio c'è”, una presa in giro di Berlusconi nella quale il nome originale, Silvio, viene sostituito con quello del Presidente della Repubblica.

Per quanto riguarda la rappresentazione dei manifestanti, si può notare come l'emittente li citi senza fare commenti o esprimere opinioni proprie ma, a differenza de l'Unità, usi qualche volta dei verbi che assumono una funzione modale nel framing delle citazioni (si veda Fairclough I995: 83 per le funzioni di questo procedimento, quali la manipolazione, e anche Halliday 2004: 252-253 per le connotazioni di vari verbi nel framing). Anche se non mette in discussione ciò che i manifestanti affermano, a volte la veridicità dei loro commenti, per 
mezzo di questo procedimento, perde di valore rispetto all'articolo de l'Unità. Ne vediamo qualche esempio:

"Noi ci sentiamo liberati", dice una signora dai capelli biondi che si definisce casalinga e mamma di due figli, uno di 27 e l'altro di 23 anni. Poi replica: "Chi siamo? Siamo gli italiani che si sono rotti di questa gentaccia, grazie a Napolitano". [...] Nella folla c'è anche un ragazzo con una decina flutes [sic!] in plastica ed una bottiglia di spumante. A lui si avvicina un altro giovane, si chiama Roberto, così dice e ha in bella mostra una bottiglia di spumante: "L'ho comperata due anni fa per questo momento", racconta.

Tramite l'uso di verbi quali "si definisce" o l'espressione "così dice", la veridicità delle affermazioni è attribuita ai parlanti, mentre l'emittente, in un certo senso, ne prende le distanze. Allo stesso tempo avviene un'individualizzazione dei manifestanti per i quali l'emittente, nel framing, adotta nuovamente dei verbi neutri come "dice", "racconta" e "replica".

Similarmente agli altri articoli, anche qui ci si occupa del lancio delle monetine. A differenza degli altri due però qui l'evento non viene né giudicato, né attenuato. L'emittente lascia la parola al leader dei Viola, senza fare commenti, sebbene adotti un verbo con connotazioni ambigue nel framing:

“Abbiamo accolto l'arrivo di Silvio Berlusconi al Quirinale con un lancio di monetine. È stato un gesto spontaneo dei cittadini. Oggi è la liberazione dell'Italia" afferma il leader dei Viola, Gianfranco Mascia, in piazza del Quirinale.

Affermare può significare sia 'sostenere' che 'confermare'; se l'emittente lo usa con il primo significato, ciò implica una presa di distanza dal commento di Mascia, mentre se lo usa attribuendogli il secondo significato, allora il framing rimane piuttosto neutro. Per quanto riguarda la rappresentazione dei manifestanti, si può notare quanto questa risulti più simile a quella dell'articolo de l'Unità rispetto a quella proposta da il Giornale. Anche qui sono descritti come dei cittadini normali, positivi nei confronti del proprio Paese e certamente non una minaccia per lo Stato: "La folla applaude al passaggio di una Cinquecento dipinta con i colori della bandiera italiana. Scatta l'applauso al Presidente della Repubblica". Qui l'emittente mette in risalto il fatto che la gente applauda due volte alla vista dei simboli che rappresentano l'Italia: i colori della bandiera e il presidente.

Nel Corriere della Sera, a differenza degli altri due giornali, mancano pareri su Berlusconi e sul suo governo da parte dell'emittente. Un'altra 
differenza è che l'emittente del Corriere dedica un intero paragrafo alla questione della fermata dell'autobus ed un altro alla situazione del traffico. Questo potrebbe essere spiegato come una volontà precisa di trasmettere informazioni di carattere pratico per il lettore comune. Per quanto riguarda la fermata, anche qui si parla della sua riapertura, come ne l'Unità, ma tutta l'informazione proviene da una lunga citazione del consigliere del Pd, Dario Nanni, in un discorso diretto, riportato senza commenti dell'emittente, ed attraverso l'uso del verbo neutro dire nel framing:

"Appena appreso che Berlusconi si sta recando al Quirinale per rassegnare le dimissioni al presidente della Repubblica ho predisposto immediatamente una mozione nella quale si chiede di ripristinare la fermata dell'autobus in via del Plebiscito" dice il consigliere capitolino del Pd Dario Nanni. "Il testo specifica - conclude - che sono venuti meno i motivi che determinarono la sospensione della fermata da parte dell'Atac su indicazione dell'am-ministrazione capitolina. Il documento sarà consegnato immediatamente agli uffici dell'assemblea capitolina per giungere lunedì stesso ad una rapida discussione della proposta".

Il linguaggio burocratico-formale di Nanni risulta oggettivo, non contenendo opinioni o parole ed espressioni con connotazioni soggettive, e costituisce inoltre un forte contrasto stilistico rispetto alla descrizione della sospensione della fermata fornita dall'articolo de l'Unità, dove il motivo sarebbe stato quello di non arrecare disturbo alle feste di Berlusconi. Nell'articolo del Corriere della Sera troviamo una perifrasi formale, "sono venuti meno i motivi che determinarono la sospensione della fermata”, mentre ne l'Unità la fermata "[e]ra stata tolta per non disturbare i balletti di Silvio".

A proposito del traffico, l'emittente riprende il discorso dal titolo e riporta informazioni sulle strade chiuse, sulle deviazioni del traffico e dei mezzi pubblici e così via. Qui individuiamo anche un ultimo esempio dell'oggettività dell'emittente, che dà la colpa della situazione del traffico sia al governo Berlusconi, sia ai manifestanti: "La crisi del governo Berlusconi ha mandato in tilt anche il traffico del centro della Capitale per le migliaia di manifestanti che si sono accalcate davanti ai palazzi del potere". Da una parte abbiamo il governo Berlusconi e la sua crisi in posizione di agente e, quindi, ritenuti responsabili dell'azione, dall'altra la subordinazione introdotta da un per causativo indica che anche i manifestanti, che qui funzionano da agente, sono una causa del problema. 


\section{Conclusioni}

I tre articoli si occupano dello stesso evento: le manifestazioni svoltesi a Roma successivamente alle dimissioni del premier Silvio Berlusconi. Sono articoli a carattere informativo da cui ci si aspetta da parte del giornalista una presentazione delle informazioni piuttosto oggettiva e priva di opinioni o simpatie personali. Nonostante gli articoli in questione dovrebbero mantenere un alto grado di oggettività in relazione all'informazione trasmessa, si può constatare che l'oggettività del giornalista/emittente varia abbastanza a seconda del profilo ideologico dei diversi quotidiani. La soggettività degli emittenti nei confronti del loro materiale può essere osservata per mezzo di diversi procedimenti linguistici. Il linguaggio offre molte possibilità di offuscare o di manipolare l'oggettività, presupposta negli articoli a carattere informativo, e ciò può avvenire a tutti i livelli del testo: dalla struttura generale alla scelta del lessico.

Analizzando i testi dei diversi giornali si può notare una grande differenza nella struttura tra il Giornale da una parte e l'Unità ed il Corriere della Sera dall'altra. Nel primo abbiamo quattro paragrafi di cui due sono di carattere retorico e due trattano delle manifestazioni e degli eventi in relazione ad esse. Ne l'Unità gli esempi di retorica soggettiva si limitano a qualche riga, mentre non vi è ombra di brani retorici nel Corriere della Sera. Gli articoli di questi due ultimi quotidiani si focalizzano spesso sui manifestanti, lasciati spesso liberi di esprimere le loro opinioni. La differenza è che l'Unità assume il punto di vista dei manifestanti, giudicando Berlusconi, mentre il Corriere della Sera mantiene la neutralità. Ne il Giornale, invece, il punto di vista dei manifestanti manca del tutto. Nell'articolo de l'Unità è tematizzata l'atmosfera di festa e di gioia dei manifestanti e in quello del Corriere della Sera ritroviamo sia l'atmosfera di festa, sia la situazione del traffico, mentre ne il Giornale è tematizzato il comportamento rimproverabile dei manifestanti. In questo modo ognuno dei quotidiani, in particolare il Giornale e l'Unità, ha dato una propria rappresentazione del mondo attraverso un suo discorso particolare.

\section{Note}

I. Per Halliday il testo è il risultato di una selezione continua in una rete di sistemi che costituiscono la grammatica di una lingua. Molte di queste scelte sono obbligatorie ed inconsce, essendo dipendenti dalla costruzione della struttura della lingua in questione (2004: 23-24). Il presente studio si concentrerà tuttavia sulle scelte consce degli emittenti. Queste si possono osservare nei contenuti 
delle tre metafunzioni del linguaggio osservate da Halliday: quella ideazionale, quella interpersonale e, infine, quella testuale. La metafunzione ideazionale (ovvero logico-esperienziale) riguarda la costruzione dell'esperienza umana e l'aspetto riflessivo della lingua. Attraverso l'analisi dei processi, dei partecipanti coinvolti e delle circostanze, si studiano la frase come rappresentazione e ciò di cui essa tratta (ivi: 29; I68-ı69; 309). La metafunzione interpersonale si occupa delle relazioni sociali e personali tra emittente e destinatario e dell'aspetto interattivo della lingua (ivi: 29-30). La metafunzione testuale riguarda la frase in quanto messaggio e la costruzione e l'organizzazione del testo (per esempio la creazione di coesione). Questa funzione ha il compito di coadiuvare le altre due metafunzioni (ivi: 30; I60). Il presente studio si focalizzerà sulla metafunzione ideazionale, cioè sui partecipanti, sui processi, sulle circostanze e sul contenuto delle frasi. Le altre due funzioni sono comunque presenti e interessanti. In particolare verrà osservata la funzione interpersonale nello studio dei tentativi, da parte dell'emittente, di influenzare il destinatario.

2. In questo studio mi occuperò esclusivamente del linguaggio del testo.

\section{Riferimenti}

\section{Corpus}

http://roma.corriere.it/notizie/cronaca/I I_novembre_I 2/dimissioni-governofesta-I902I/4602005 I.shtml

http://cerca.unita.it/ARCHIVE/xml/2335000/23340I9-xml?key?=bandiere $\%{ }_{2} \mathrm{C}+$ musica $\%{ }_{2} \mathrm{C}+$ cori \&first $=\mathrm{I} \&$ orderby $=\mathrm{I}$

http://www.ilgiornale.it/interni/eccola_italia_sfascisticarosellidimissio$\mathrm{ni} / \mathrm{I} 2-\mathrm{II}-200 \mathrm{I} \mathrm{I} /$ articolo-id $=5563 \mathrm{I}$-page $=0-$ comments $=\mathrm{I}$

\section{Opere citate}

Fairclough, Norman. 1995. Media Discourse. London: Edward Arnold.

Fairclough, Norman. 2003. Analysing Discourse. Textual analysis for social research. London \& New York: Routledge.

Fowler, Roger. I991. Language in the News. Discourse and Ideology in the Press. London \& New York: Routledge.

Halliday, M.A.K. 2004. An Introduction to Functional Grammar. (Revised by Christian M.I.M Matthiessen). London: Hodder Education.

Searle, John. 1979. Expression and Meaning. Studies in the Theory of Speech Acts. Cambridge: Cambridge UP.

Lo Zingarelli. Vocabolario della lingua italiana di Nicola Zingarelli. 1996. Dodicesima edizione. Bologna: Zanichelli. 